\title{
Article \\ On Some New Weighted Steffensen-Type Inequalities on Time Scales
}

\author{
Ahmed A. El-Deeb ${ }^{1, *}$, Omar Bazighifan ${ }^{2}$ (D) and Jan Awrejcewicz ${ }^{3, *}$ (D) \\ 1 Department of Mathematics, Faculty of Science, Al-Azhar University, Nasr City, Cairo 11884, Egypt \\ 2 Section of Mathematics, International Telematic University Uninettuno, Corso Vittorio Emanuele II, 39, \\ 00186 Rome, Italy; o.bazighifan@gmail.com \\ 3 Department of Automation, Biomechanics and Mechatronics, Lodz University of Technology, \\ 1/15 Stefanowski St., 90-924 Lodz, Poland \\ * Correspondence: ahmedeldeeb@azhar.edu.eg (A.A.E.-D.); jan.awrejcewicz@p.lodz.pl (J.A.)
}

check for updates

Citation: El-Deeb, A.A.; Bazighifan, O.; Awrejcewicz, J. On Some New Weighted Steffensen-Type Inequalities on Time Scales. Mathematics 2021, 9, 2670. https://doi.org/10.3390/ math9212670

Academic Editor: Wei-Shih Du

Received: 18 September 2021 Accepted: 15 Ocotober 2021 Published: 21 Ocotober 2021

Publisher's Note: MDPI stays neutral with regard to jurisdictional claims in published maps and institutional affiliations.

Copyright: (c) 2020 by the authors. Licensee MDPI, Basel, Switzerland. This article is an open access article distributed under the terms and conditions of the Creative Commons Attribution (CC BY) license (https:// creativecommons.org/licenses/by/ $4.0 /)$.
Abstract: The motivation of this paper is to explore some new inequalities of Steffensen-type which were demonstrated by Pečarić and Kalamir in 2014. The main idea is to investigate a class of certain inequalities by employing diamond- $\alpha$ dynamic integral on time scales. In addition, to obtain some new inequalities as special cases, we also extend our results to continuous and discrete calculations.

Keywords: diamond- $\alpha$ dynamic integral; Steffensen's inequality; dynamic inequality; time scale

\section{Introduction}

In 2014, Pečarić and Kalamir in [1] have established the following interesting theorem:

Theorem 1. Let $h:[a, b] \rightarrow \mathbb{R}$ be a positive integrable function, $f:[a, b] \rightarrow \mathbb{R}$ be an integrable function, and let $c \in(a, b)$. Further, assume that $g:[a, b] \rightarrow \mathbb{R}$ is integrable function such that $0 \leq g(t) \leq 1$ for all $t \in[a, b]$. Moreover, let $\lambda_{1}$ be the solution of the equation

$$
\int_{a}^{a+\lambda_{1}} h(t) d t=\int_{a}^{c} h(t) g(t) d t
$$

and $\lambda_{2}$ be the solution of the equation

$$
\int_{b-\lambda_{2}}^{b} h(t) d t=\int_{c}^{b} h(t) g(t) d t
$$

If there exists a constant $A$ such that $f(t) / h(t)-A t$ is nonincreasing on $[a, c]$ and nondecreasing on $[c, b]$, and

$$
\int_{a}^{b} t h(t) g(t) d t=\int_{a}^{a+\lambda_{1}} t h(t) d t+\int_{b-\lambda_{2}}^{b} t h(t) d t
$$

then

$$
\int_{a}^{b} f(t) g(t) d t \leq \int_{a}^{a+\lambda_{1}} f(t) d t+\int_{b-\lambda_{2}}^{b} f(t) d t .
$$

In 1988, Stefan Hilger [2] initiated the theory of time scales in their PhD thesis [3] in order to unify discrete and continuous analysis. The book by Bohner and Peterson [4] on the subject of time scales briefs and organizes much of time scales calculus. 
A time scale $\mathbb{T}$ is an arbitrary nonempty closed subset of the set of real numbers $\mathbb{R}$. Throughout the article, we assume that $\mathbb{T}$ has the topology that it inherits from the standard topology on $\mathbb{R}$. We define the forward jump operator $\sigma: \mathbb{T} \rightarrow \mathbb{T}$ for any $t \in \mathbb{T}$ by

$$
\sigma(t):=\inf \{s \in \mathbb{T}: s>t\}
$$

and the backward jump operator $\rho: \mathbb{T} \rightarrow \mathbb{T}$ for any $t \in \mathbb{T}$ by

$$
\rho(t):=\sup \{s \in \mathbb{T}: s<t\} \text {. }
$$

In the preceding two definitions, we set $\inf \varnothing=\sup \mathbb{T}$ (i.e., if $t$ is the maximum of $\mathbb{T}$, then $\sigma(t)=t$ ) and $\sup \varnothing=\inf \mathbb{T}$ (i.e., if $t$ is the minimum of $\mathbb{T}$, then $\rho(t)=t$ ), where $\varnothing$ denotes the empty set.

A point $t \in \mathbb{T}$ with inf $\mathbb{T}<t<\sup \mathbb{T}$ is said to be right-scattered if $\sigma(t)>t$, rightdense if $\sigma(t)=t$, left-scattered if $\rho(t)<t$, and left-dense if $\rho(t)=t$. Points that are simultaneously right-dense and left-dense are said to be dense points, whereas points that are simultaneously right-scattered and left-scattered are said to be isolated points.

The forward graininess function $\mu: \mathbb{T} \rightarrow[0, \infty)$ is defined for any $t \in \mathbb{T}$ by $\mu(t):=\sigma(t)-t$.

If $f: \mathbb{T} \rightarrow \mathbb{R}$ is a function, then the function $f^{\sigma}: \mathbb{T} \rightarrow \mathbb{R}$ is defined by $f^{\sigma}(t)=f(\sigma(t)), \forall t \in \mathbb{T}$, that is $f^{\sigma}=f \circ \sigma$. Similarly, the function $f^{\rho}: \mathbb{T} \rightarrow \mathbb{R}$ is defined by $f^{\rho}(t)=g(\rho(t)), \forall t \in \mathbb{T}$, that is $f^{\rho}=f \circ \rho$.

The sets $\mathbb{T}^{\kappa}, \mathbb{T}_{\kappa}$ and $\mathbb{T}_{\kappa}^{\kappa}$ are introduced as follows: If $\mathbb{T}$ has a left-scattered maximum $t_{1}$, then $\mathbb{T}^{\kappa}=\mathbb{T}-\left\{t_{1}\right\}$, otherwise $\mathbb{T}^{\mathcal{K}}=\mathbb{T}$. If $\mathbb{T}$ has a right-scattered minimum $t_{2}$, then $\mathbb{T}_{\mathcal{K}}=\mathbb{T}-\left\{t_{2}\right\}$, otherwise $\mathbb{T}_{\kappa}=\mathbb{T}$. Finally, we have $\mathbb{T}_{\mathcal{K}}^{\kappa}=\mathbb{T}^{\mathcal{K}} \cap \mathbb{T}_{\mathcal{\kappa}}$.

The interval $[a, b]$ in $\mathbb{T}$ is defined by

$$
[a, b]_{\mathbb{T}}=\{t \in \mathbb{T}: a \leq t \leq b\}
$$

We define the open intervals and half-closed intervals similarly.

We will use the following crucial relations between calculus on time scales $\mathbb{T}$ and either differential calculus on $\mathbb{R}$ or difference calculus on $\mathbb{Z}$. Note that:

(i) If $\mathbb{T}=\mathbb{R}$, then

$$
\begin{gathered}
\sigma(t)=\rho(t)=t, \quad \mu(t)=v(t)=0, \quad f^{\Delta}(t)=f^{\nabla}(t)=f^{\prime}(t), \\
\int_{a}^{b} f(t) \Delta t=\int_{a}^{b} f(t) \nabla t=\int_{a}^{b} f(t) d t .
\end{gathered}
$$

(ii) If $\mathbb{T}=\mathbb{Z}$, then

$$
\begin{gathered}
\sigma(t)=t+1, \quad \rho(t)=t-1, \quad \mu(t)=v(t)=1, \\
f^{\Delta}(t)=\Delta f(t), \quad f^{\nabla}(t)=\nabla f(t), \\
\int_{a}^{b} f(t) \Delta t=\sum_{t=a}^{b-1} f(t), \quad \int_{a}^{b} f(t) \nabla t=\sum_{t=a+1}^{b} f(t),
\end{gathered}
$$

where $\Delta$ and $\nabla$ are the forward and backward difference operators, respectively.

the diamond- $\alpha$ dynamic derivative of $f$ at $t$ is defined by

$$
f^{\nabla^{\alpha}}(t)=\alpha f^{\Delta}(t)+(1-\alpha) f^{\nabla}(t), \quad 0 \leq \alpha \leq 1 .
$$

For more details on the diamond- $\alpha$ calculus on time scales, we refer the interested reader to [5].

Over the past decade, a great number of dynamic inequalities on time scales has been established by many researchers who were motivated by some applications (see [4,6-11]). Some researchers developed various results concerning fractional calculus on time scales to produce related dynamic inequalities (see [12-15]). 
Anderson [16] was the first to extend the Steffensen inequality to a general time scale. In particular, he gave the following result.

Theorem 2. Suppose that $a, b \in \mathbb{T}_{\kappa}$ with $a<b$, and $f, g:[a, b]_{\mathbb{T}} \rightarrow \mathbb{R}$ are $\nabla$-integrable functions such that $f$ is of one sign and nonincreasing and $0 \leq g(t) \leq 1$ on $[a, b]_{\mathbb{T}}$. Further, assume that $\lambda=\int_{a}^{b} g(t) \nabla t$ such that $b-\lambda, a+\lambda \in \mathbb{T}$. Then

$$
\int_{b-\lambda}^{b} f(t) \nabla t \leq \int_{a}^{b} f(t) g(t) \nabla t \leq \int_{a}^{a+\lambda} f(t) \nabla t .
$$

In [17], Özkan and Yildirim established the following results regarding diamond- $\alpha$ dynamic Steffensen-type inequalities.

Theorem 3. Let $h$ be a positive integrable function on $[a, b]_{\mathbb{T}}$ and $f, g$ be integrable functions on $[a, b]_{\mathbb{T}}$ such that $f$ is nonincreasing and $0 \leq g(t) \leq h(t)$ for all $t \in[a, b]_{\mathbb{T}}$. Then

$$
\int_{a}^{b} f(t) g(t) \diamond_{\alpha} t \leq \int_{a}^{a+\lambda} f(t) h(t) \diamond_{\alpha} t
$$

where $\lambda$ is the solution of the equation

$$
\int_{a}^{b} g(t) \diamond_{\alpha} t=\int_{a}^{a+\lambda} h(t) \diamond_{\alpha} t
$$

If $f / h$ is nondecreasing, then the reverse inequality in (4) holds.

Theorem 4. Let $h$ be a positive integrable function on $[a, b]_{\mathbb{T}}$ and $f, g$ be integrable functions on $[a, b]_{\mathbb{T}}$ such that $f$ is nonincreasing and $0 \leq g(t) \leq h(t)$ for all $t \in[a, b]_{\mathbb{T}}$. Then

$$
\int_{b-\lambda}^{b} f(t) h(t) \diamond_{\alpha} t \leq \int_{a}^{b} f(t) g(t) \diamond_{\alpha} t
$$

where $\lambda$ is the solution of the equation

$$
\int_{b-\lambda}^{b} h(t) \diamond_{\alpha} t=\int_{a}^{b} g(t) \diamond_{\alpha} t .
$$

If $f / h$ is nondecreasing, then the reverse inequality in (5) holds.

Theorem 5. Let $h$ be a positive integrable function on $[a, b]_{\mathbb{T}}$ and $f, g, \psi$ be integrable functions on $[a, b]_{\mathbb{T}}$ such that $f$ is nonincreasing and $0 \leq \psi(t) \leq g(t) \leq h(t)-\psi(t)$ for all $t \in[a, b]_{\mathbb{T}}$. Then

$$
\begin{aligned}
& \int_{b-\lambda}^{b} f(t) h(t) \diamond_{\alpha} t+\int_{a}^{b}|(f(t)-f(b-\lambda)) \psi(t)| \nabla_{\alpha} t \\
& \leq \int_{a}^{b} f(t) g(t) \diamond_{\alpha} t \\
& \leq \int_{a}^{a+\lambda} f(t) h(t) \nabla_{\alpha} t-\int_{a}^{b}|(f(t)-f(a+\lambda)) \psi(t)| \nabla_{\alpha} t,
\end{aligned}
$$

where $\lambda$ is the solution of the equation

$$
\int_{a}^{a+\lambda} h(t) \diamond_{\alpha} t=\int_{a}^{b} g(t) \diamond_{\alpha} t=\int_{b-\lambda}^{b} h(t) \diamond_{\alpha} t .
$$


Theorem 6. Let $f, g$ and $h$ be $\diamond_{\alpha}$-integrable functions defined on $[a, b]_{\mathbb{T}}$ with $f$ nonincreasing. Furthermore, let $0 \leq g(t) \leq h(t)$ for all $t \in[a, b]_{\mathbb{T}}$. Then

$$
\begin{aligned}
\int_{b-\lambda}^{b} f(t) h(t) \diamond_{\alpha} t & \leq \int_{b-\lambda}^{b}[f(t) h(t)-(f(t)-f(b-\lambda))][h(t)-g(t)] \nabla_{\alpha} t \\
& \leq \int_{a}^{b} f(t) g(t) \diamond_{\alpha} t \\
& \leq \int_{a}^{a+\lambda}[f(t) h(t)-(f(t)-f(a+\lambda))][h(t)-g(t)] \nabla_{\alpha} t \\
& \leq \int_{a}^{a+\lambda} f(t) h(t) \nabla_{\alpha} t
\end{aligned}
$$

where $\lambda$ is given by

$$
\int_{a}^{a+\lambda} h(t) \diamond_{\alpha} t=\int_{a}^{b} g(t) \diamond_{\alpha} t=\int_{b-\lambda}^{b} h(t) \diamond_{\alpha} t .
$$

In this paper, we extend some generalizations of integral Steffensen's inequality given in [1] to a general time scale, and establish several new sharpened versions of diamond- $\alpha$ dynamic Steffensen's inequality on time scales. As special cases of our results, we recover the integral inequalities given in these papers. Our results also give some new discrete Steffensen's inequalities. We obtain the new dynamic Steffensen inequalities using the diamond- $\alpha$ integrals on time scales. For $\alpha=1$, the diamond- $\alpha$ integral becomes delta integral and for $\alpha=0$ it becomes nabla integral.

Now, we are ready to state and prove the main results of this paper.

\section{Main Results}

Let us begin by introducing a class of functions that extends the class of convex functions.

Definition 1. Let $\phi, h:[a, b]_{\mathbb{T}} \rightarrow \mathbb{R}$ be positive functions, $f:[a, b]_{\mathbb{T}} \rightarrow \mathbb{R}$ be a function, and $c \in(a, b)$. We say that $f^{\sigma} / h$ belongs to the class $\mathbb{A H}_{1}^{c}[a, b]$ (respectively, $\mathbb{A H}_{2}^{c}[a, b]$ ) if there exists a constant $A$ such that the function $f^{\sigma}(t) / h(t)-A \phi(t)$ is nonincreasing (respectively, nondecreasing) on $[a, c]_{\mathbb{T}}$ and nondecreasing (respectively, nonincreasing) on $[c, b]_{\mathbb{T}}$.

We shall need the following lemmas in the proof of our results.

Lemma 1. Let $h$ be a positive integrable function on $[a, b]_{\mathbb{T}}$ and $f, g$ be integrable functions on $[a, b]_{\mathbb{T}}$ such that $f / h$ is nonincreasing and $0 \leq g(t) \leq 1$ for all $t \in[a, b]_{\mathbb{T}}$. Then

$$
\int_{a}^{b} f(t) g(t) \diamond_{\alpha} t \leq \int_{a}^{a+\lambda} f(t) \diamond_{\alpha} t,
$$

where $\lambda$ is the solution of the equation

$$
\int_{a}^{b} h(t) g(t) \diamond_{\alpha} t=\int_{a}^{a+\lambda} h(t) \diamond_{\alpha} t .
$$

If $f / h$ is nondecreasing, then the reverse inequality in (6) holds.

Proof. Take $g(t) \mapsto h(t) g(t)$ and $f(t) \mapsto f(t) / h(t)$ in Theorem 3.

Lemma 2. Let $h$ be a positive integrable function on $[a, b]_{\mathbb{T}}$ and $f, g$ be integrable functions on $[a, b]_{\mathbb{T}}$ such that $f / h$ is nonincreasing and $0 \leq g(t) \leq 1$ for all $t \in[a, b]_{\mathbb{T}}$. Then

$$
\int_{b-\lambda}^{b} f(t) \diamond_{\alpha} t \leq \int_{a}^{b} f(t) g(t) \diamond_{\alpha} t
$$


where $\lambda$ is the solution of the equation

$$
\int_{b-\lambda}^{b} h(t) \diamond_{\alpha} t=\int_{a}^{b} h(t) g(t) \diamond_{\alpha} t
$$

If $f / h$ is nondecreasing, then the reverse inequality in (7) holds.

Proof. Take $g(t) \mapsto h(t) g(t)$ and $f(t) \mapsto f(t) / h(t)$ in Theorem 4 .

Lemma 3. Let $h$ be a positive integrable function on $[a, b]_{\mathbb{T}}$ and $f, g, \psi$ be integrable functions on $[a, b]_{\mathbb{T}}$ such that $f / h$ is nonincreasing and $0 \leq \psi(t) \leq g(t) \leq 1-\psi(t)$ for all $t \in[a, b]_{\mathbb{T}}$. Then

$$
\begin{aligned}
& \int_{b-\lambda}^{b} f(t) \diamond_{\alpha} t+\int_{a}^{b}\left|\left(\frac{f(t)}{h(t)}-\frac{f(b-\lambda)}{h(b-\lambda)}\right) h(t) \psi(t)\right| \nabla_{\alpha} t \\
& \leq \int_{a}^{b} f(t) g(t) \nabla_{\alpha} t \\
& \leq \int_{a}^{a+\lambda} f(t) \nabla_{\alpha} t-\int_{a}^{b}\left|\left(\frac{f(t)}{h(t)}-\frac{f(a+\lambda)}{h(a+\lambda)}\right) h(t) \psi(t)\right| \nabla_{\alpha} t
\end{aligned}
$$

where $\lambda$ is the solution of the equation

$$
\int_{a}^{a+\lambda} h(t) \diamond_{\alpha} t=\int_{a}^{b} h(t) g(t) \diamond_{\alpha} t=\int_{b-\lambda}^{b} h(t) \diamond_{\alpha} t .
$$

Proof. Take $g(t) \mapsto h(t) g(t), f(t) \mapsto f(t) / h(t)$ and $\psi(t) \mapsto h(t) \psi(t)$ in Theorem 5 .

Lemma 4. Let $h$ be a positive $\diamond_{\alpha}$-integrable function on $[a, b]_{\mathbb{T}}$, and $f, g$ be $\diamond_{\alpha}$-integrable functions on $[a, b]_{\mathbb{T}}$, Further, assume that $f / h$ is nonincreasing and $0 \leq g(t) \leq 1$ for all $t \in[a, b]_{\mathbb{T}}$. Then

$$
\begin{aligned}
\int_{b-\lambda}^{b} f(t) \diamond_{\alpha} t & \leq \int_{b-\lambda}^{b}\left(f(t)-\left[\frac{f(t)}{h(t)}-\frac{f(b-\lambda)}{h(b-\lambda)}\right] h(t)[1-g(t)]\right) \nabla_{\alpha} t \\
& \leq \int_{a}^{b} f(t) g(t) \nabla_{\alpha} t \\
& \leq \int_{a}^{a+\lambda}\left(f(t)-\left[\frac{f(t)}{h(t)}-\frac{f(a+\lambda)}{h(a+\lambda)}\right] h(t)[1-g(t)]\right) \nabla_{\alpha} t \\
& \leq \int_{a}^{a+\lambda} f(t) \diamond_{\alpha} t
\end{aligned}
$$

where $\lambda$ is given by

$$
\int_{a}^{a+\lambda} h(t) \diamond_{\alpha} t=\int_{a}^{b} h(t) g(t) \nabla_{\alpha} t=\int_{b-\lambda}^{b} h(t) \diamond_{\alpha} t .
$$

Proof. Take $g(t) \mapsto h(t) g(t)$ and $f(t) \mapsto f(t) / h(t)$ in Theorem 6 .

Theorem 7. Let $h:[a, b]_{\mathbb{T}} \rightarrow \mathbb{R}$ be a positive $\diamond_{\alpha}$-integrable function, let $f:[a, b]_{\mathbb{T}} \rightarrow \mathbb{R}$ be $a$ $\diamond_{\alpha}$-integrable function, and let $c \in(a, b)$. Let $g:[a, b]_{\mathbb{T}} \rightarrow \mathbb{R}$ be $a \diamond_{\alpha}$-integrable function such that $0 \leq g(t) \leq 1$ for all $t \in[a, b]_{\mathbb{T}}$. Let $\lambda_{1}$ be the solution of the equation

$$
\int_{a}^{a+\lambda_{1}} h(t) \diamond_{\alpha} t=\int_{a}^{c} h(t) g(t) \diamond_{\alpha} t
$$

and let $\lambda_{2}$ be the solution of the equation

$$
\int_{b-\lambda_{2}}^{b} h(t) \diamond_{\alpha} t=\int_{c}^{b} h(t) g(t) \diamond_{\alpha} t .
$$


If $f^{\sigma} / h \in \mathbb{A H}_{1}^{c}[a, b]$ and

$$
\int_{a}^{b} \phi(t) h(t) g(t) \diamond_{\alpha} t=\int_{a}^{a+\lambda_{1}} \phi(t) h(t) \diamond_{\alpha} t+\int_{b-\lambda_{2}}^{b} \phi(t) h(t) \diamond_{\alpha} t,
$$

then

$$
\int_{a}^{b} f^{\sigma}(t) g(t) \diamond_{\alpha} t \leq \int_{a}^{a+\lambda_{1}} f^{\sigma}(t) \diamond_{\alpha} t+\int_{b-\lambda_{2}}^{b} f^{\sigma}(t) \diamond_{\alpha} t .
$$

If $f^{\sigma} / h \in \mathbb{A H}_{2}^{c}[a, b]$ and (8) holds, the inequality in (9) is reversed.

Proof. Let $f^{\sigma} / h \in \mathbb{A H}_{1}^{c}[a, b]$, and let $F(x)=f^{\sigma}(x)-A \phi(x) h(x)$, where $A$ is the constant from Definition 1 . Since $F / h:[a, c]_{\mathbb{T}} \rightarrow \mathbb{R}$ is nonincreasing, from Lemma 1 we obtain

$$
\begin{aligned}
0 \leq & \int_{a}^{a+\lambda_{1}} F(t) \diamond_{\alpha} t-\int_{a}^{c} F(t) g(t) \diamond_{\alpha} t \\
= & \int_{a}^{a+\lambda_{1}} f^{\sigma}(t) \diamond_{\alpha} t-\int_{a}^{c} f^{\sigma}(t) g(t) \diamond_{\alpha} t \\
& -A\left(\int_{a}^{a+\lambda_{1}} \phi(t) h(t) \diamond_{\alpha} t-\int_{a}^{c} \phi(t) h(t) g(t) \diamond_{\alpha} t\right) .
\end{aligned}
$$

Since $F / h:[c, b]_{\mathbb{T}} \rightarrow \mathbb{R}$ is nondecreasing, from Lemma 2 we obtain

$$
\begin{aligned}
0 \geq & \int_{c}^{b} F(t) g(t) \diamond_{\alpha} t-\int_{b-\lambda_{2}}^{b} F(t) \diamond_{\alpha} t \\
= & \int_{c}^{b} f^{\sigma}(t) g(t) \diamond_{\alpha} t-\int_{b-\lambda_{2}}^{b} f^{\sigma}(t) \diamond_{\alpha} t \\
& -A\left(\int_{c}^{b} \phi(t) h(t) g(t) \diamond_{\alpha} t-\int_{b-\lambda_{2}}^{b} \phi(t) h(t) \diamond_{\alpha} t\right) .
\end{aligned}
$$

Now, from (10) and (11) we obtain

$$
\begin{aligned}
& \int_{a}^{a+\lambda_{1}} f^{\sigma}(t) \diamond_{\alpha} t+\int_{b-\lambda_{2}}^{b} f^{\sigma}(t) \diamond_{\alpha} t-\int_{a}^{b} f^{\sigma}(t) g(t) \diamond_{\alpha} t \\
& \geq A\left(\int_{a}^{a+\lambda_{1}} \phi(t) h(t) \diamond_{\alpha} t+\int_{b-\lambda_{2}}^{b} \phi(t) h(t) \diamond_{\alpha} t-\int_{a}^{b} \phi(t) h(t) g(t) \diamond_{\alpha} t\right)
\end{aligned}
$$

Hence, if (8) is satisfied, then (9) holds. It is similar for $f^{\sigma} / h \in \mathbb{A H}_{2}^{c}[a, b]$.

Corollary 1. Setting $\alpha=1$ in Theorem 7, we obtain the delta version of inequality (9) as follows:

$$
\int_{a}^{b} f^{\sigma}(t) g(t) \Delta t \leq \int_{a}^{a+\lambda_{1}} f^{\sigma}(t) \Delta t+\int_{b-\lambda_{2}}^{b} f^{\sigma}(t) \Delta t .
$$

Corollary 2. Setting $\alpha=0$ in Theorem 7, we obtain the nabla version of inequality (9) as follows:

$$
\int_{a}^{b} f^{\sigma}(t) g(t) \nabla t \leq \int_{a}^{a+\lambda_{1}} f^{\sigma}(t) \nabla t+\int_{b-\lambda_{2}}^{b} f^{\sigma}(t) \nabla t .
$$

Corollary 3. If $\mathbb{T}=\mathbb{R}$ and $\phi(t)=t$ in Corollary 1, then, with the help of relations (1), we recapture [1] (Theorem 8 ).

Corollary 4. If $\mathbb{T}=\mathbb{Z}$ in Corollary 1, then, with the help of relations (2), inequality (12) becomes

$$
\sum_{t=a}^{b-1} f(t+1) g(t) \leq \sum_{t=a}^{a+\lambda_{1}-1} f(t+1)+\sum_{t=b-\lambda_{2}}^{b-1} f(t+1) .
$$


Theorem 8. Let $h:[a, b]_{\mathbb{T}} \rightarrow \mathbb{R}$ be a positive $\diamond_{\alpha}$-integrable function, let $f:[a, b]_{\mathbb{T}} \rightarrow \mathbb{R}$ be a $\diamond_{\alpha}$-integrable function, and let $c \in(a, b)$. Let $g:[a, b]_{\mathbb{T}} \rightarrow \mathbb{R}$ be $a \diamond_{\alpha}$-integrable function such that $0 \leq g(t) \leq 1$ for all $t \in[a, b]_{\mathbb{T}}$. Let $\lambda_{1}$ be the solution of the equation

$$
\int_{\mathcal{c}-\lambda_{1}}^{c} h(t) \diamond_{\alpha} t=\int_{a}^{c} h(t) g(t) \diamond_{\alpha} t
$$

and let $\lambda_{2}$ be the solution of the equation

$$
\int_{c}^{c+\lambda_{2}} h(t) \diamond_{\alpha} t=\int_{c}^{b} h(t) g(t) \diamond_{\alpha} t
$$

If $f^{\sigma} / h \in \mathbb{A H}_{1}^{c}[a, b]$ and

$$
\int_{a}^{b} \phi(t) h(t) g(t) \diamond_{\alpha} t=\int_{c-\lambda_{1}}^{c+\lambda_{2}} \phi(t) h(t) \diamond_{\alpha} t,
$$

then

$$
\int_{a}^{b} f^{\sigma}(t) g(t) \diamond_{\alpha} t \geq \int_{\mathcal{c}-\lambda_{1}}^{c+\lambda_{2}} f^{\sigma}(t) \diamond_{\alpha} t
$$

If $f^{\sigma} / h \in \mathbb{A H}_{2}^{c}[a, b]$ and (15) holds, the inequality in (16) is reversed.

Proof. Let $f^{\sigma} / h \in \mathbb{A H}_{1}^{c}[a, b]$, and let $F(x)=f^{\sigma}(x)-A \phi(x) h(x)$, where $A$ is the constant from Definition 1. $F / h:[a, c]_{\mathbb{T}} \rightarrow \mathbb{R}$ is nonincreasing, so from Lemma 1 we obtain

$$
\begin{aligned}
0 \leq & \int_{a}^{c} f^{\sigma}(t) g(t) \diamond_{\alpha} t-\int_{c-\lambda_{1}}^{c} f^{\sigma}(t) \diamond_{\alpha} t \\
& -A\left(\int_{a}^{c} \phi(t) h(t) g(t) \diamond_{\alpha} t-\int_{c-\lambda_{1}}^{c} \phi(t) h(t) \diamond_{\alpha} t\right) .
\end{aligned}
$$

$F / h:[c, b]_{\mathbb{T}} \rightarrow \mathbb{R}$ is nondecreasing, so from Lemma 1 we obtain

$$
\begin{aligned}
0 \geq & \int_{c}^{c+\lambda_{2}} f^{\sigma}(t) \diamond_{\alpha} t-\int_{c}^{b} f^{\sigma}(t) g(t) \nabla_{\alpha} t \\
& -A\left(\int_{\mathcal{c}}^{c+\lambda_{2}} \phi(t) h(t) \diamond_{\alpha} t-\int_{c}^{b} \phi(t) h(t) g(t) \diamond_{\alpha} t\right) .
\end{aligned}
$$

Hence, from (17) and (18) we obtain

$$
\begin{aligned}
& \int_{a}^{b} f^{\sigma}(t) g(t) \diamond_{\alpha} t-\int_{\mathcal{c}-\lambda_{1}}^{c+\lambda_{2}} f^{\sigma}(t) \diamond_{\alpha} t \\
& \geq A\left(\int_{a}^{b} \phi(t) h(t) g(t) \diamond_{\alpha} t-\int_{\mathcal{c}-\lambda_{1}}^{c+\lambda_{2}} \phi(t) h(t) \diamond_{\alpha} t\right)
\end{aligned}
$$

Hence, if $\int_{a}^{b} \phi(t) h(t) g(t) \diamond_{\alpha} t=\int_{c-\lambda_{1}}^{c+\lambda_{2}} \phi(t) h(t) \diamond_{\alpha} t$ is satisfied, then (16) holds. It is similar for $f^{\sigma} / h \in \mathbb{A H}_{2}^{c}[a, b]$.

Corollary 5. Setting $\alpha=1$ in Theorem 8, we obtain the delta version of inequality (16) as follows:

$$
\int_{a}^{b} f^{\sigma}(t) g(t) \Delta t \geq \int_{c-\lambda_{1}}^{c+\lambda_{2}} f^{\sigma}(t) \Delta t .
$$

Corollary 6. Setting $\alpha=0$ in Theorem 8, we obtain the nabla version of inequality (16) as follows:

$$
\int_{a}^{b} f^{\sigma}(t) g(t) \nabla t \geq \int_{\mathcal{c}-\lambda_{1}}^{c+\lambda_{2}} f^{\sigma}(t) \nabla t .
$$


Corollary 7. If $\mathbb{T}=\mathbb{R}$ and $\phi(t)=t$ in Corollary 5, then, with the help of relations (1), we recapture [1] (Theorem 10).

Corollary 8. If $\mathbb{T}=\mathbb{Z}$ in Corollary 5 , then, with the help of relations (2), inequality (19) becomes

$$
\sum_{t=a}^{b-1} f(t+1) g(t) \geq \sum_{t=c-\lambda_{1}}^{c+\lambda_{2}-1} f(t+1) .
$$

Theorem 9. Let $h:[a, b]_{\mathbb{T}} \rightarrow \mathbb{R}$ be a positive $\diamond_{\alpha}$-integrable function, let $f:[a, b]_{\mathbb{T}} \rightarrow \mathbb{R}$ be a $\diamond_{\alpha}$-integrable function, and let $c \in(a, b)$. Let $g:[a, b]_{\mathbb{T}} \rightarrow \mathbb{R}$ be $a \diamond_{\alpha}$-integrable function such that $0 \leq g(t) \leq h(t)$ for all $t \in[a, b]_{\mathbb{T}}$. Let $\lambda_{1}$ be the solution of the equation

$$
\int_{a}^{a+\lambda_{1}} h(t) \diamond_{\alpha} t=\int_{a}^{c} g(t) \diamond_{\alpha} t
$$

and let $\lambda_{2}$ be the solution of the equation

$$
\int_{b-\lambda_{2}}^{b} h(t) \diamond_{\alpha} t=\int_{c}^{b} g(t) \diamond_{\alpha} t
$$

If $f^{\sigma} / h \in \mathbb{A H}_{1}^{c}[a, b]$ and

$$
\int_{a}^{b} \phi(t) g(t) \diamond_{\alpha} t=\int_{a}^{a+\lambda_{1}} \phi(t) h(t) \diamond_{\alpha} t+\int_{b-\lambda_{2}}^{b} \phi(t) h(t) \diamond_{\alpha} t,
$$

then

$$
\int_{a}^{b} f^{\sigma}(t) g(t) \diamond_{\alpha} t \leq \int_{a}^{a+\lambda_{1}} f^{\sigma}(t) h(t) \diamond_{\alpha} t+\int_{b-\lambda_{2}}^{b} f^{\sigma}(t) h(t) \diamond_{\alpha} t .
$$

If $f^{\sigma} / h \in \mathbb{A H}_{2}^{c}[a, b]$ and (22) holds, the inequality in (23) is reversed.

Proof. Take $g \mapsto g / h$ and $f \mapsto f h$ in Theorem 7 .

Corollary 9. Setting $\alpha=1$ in Theorem 9, we obtain the delta version of inequality (23) as follows:

$$
\int_{a}^{b} f^{\sigma}(t) g(t) \Delta t \leq \int_{a}^{a+\lambda_{1}} f^{\sigma}(t) h(t) \Delta t+\int_{b-\lambda_{2}}^{b} f^{\sigma}(t) h(t) \Delta t .
$$

Corollary 10. Setting $\alpha=0$ in Theorem 9, we obtain the nabla version of inequality (23) as follows:

$$
\int_{a}^{b} f^{\sigma}(t) g(t) \nabla t \leq \int_{a}^{a+\lambda_{1}} f^{\sigma}(t) h(t) \nabla t+\int_{b-\lambda_{2}}^{b} f^{\sigma}(t) h(t) \nabla t
$$

Corollary 11. If $\mathbb{T}=\mathbb{R}$ and $\phi(t)=t$ in Corollary 9, then, with the help of relations (1), we recapture [1] (Theorem 16).

Corollary 12. If $\mathbb{T}=\mathbb{Z}$ in Corollary 9, then, with the help of relations (2), inequality (24) becomes

$$
\sum_{t=a}^{b-1} f(t+1) g(t) \leq \sum_{t=a}^{a+\lambda_{1}-1} f(t+1) h(t)+\sum_{t=b-\lambda_{2}}^{b-1} f(t+1) h(t) .
$$


Theorem 10. Let $h:[a, b]_{\mathbb{T}} \rightarrow \mathbb{R}$ be a positive $\diamond_{\alpha}$-integrable function, let $f:[a, b]_{\mathbb{T}} \rightarrow \mathbb{R}$ be a $\nabla_{\alpha}$-integrable function, and let $c \in(a, b)$. Let $g:[a, b]_{\mathbb{T}} \rightarrow \mathbb{R}$ be $a \diamond_{\alpha}$-integrable function such that $0 \leq g(t) \leq h(t)$ for all $t \in[a, b]_{\mathbb{T}}$. Let $\lambda_{1}$ be the solution of the equation

$$
\int_{c-\lambda_{1}}^{c} h(t) \diamond_{\alpha} t=\int_{a}^{c} g(t) \diamond_{\alpha} t
$$

and let $\lambda_{2}$ be the solution of the equation

$$
\int_{\mathcal{c}}^{c+\lambda_{2}} h(t) \diamond_{\alpha} t=\int_{c}^{b} g(t) \diamond_{\alpha} t
$$

If $f^{\sigma} / h \in \mathbb{A H}_{1}^{c}[a, b]$ and

$$
\int_{a}^{b} \phi(t) g(t) \diamond_{\alpha} t=\int_{c-\lambda_{1}}^{c+\lambda_{2}} \phi(t) h(t) \diamond_{\alpha} t
$$

then

$$
\int_{a}^{b} f^{\sigma}(t) g(t) \diamond_{\alpha} t \geq \int_{c-\lambda_{1}}^{c+\lambda_{2}} f^{\sigma}(t) h(t) \diamond_{\alpha} t .
$$

If $f^{\sigma} / h \in \mathbb{A H}_{2}^{c}[a, b]$ and (27) holds, the inequality in (28) is reversed.

Proof. Take $g \mapsto g / h$ and $f \mapsto f h$ in Theorem 8 .

Corollary 13. Setting $\alpha=1$ in Theorem 10, we obtain the delta version of inequality (28) as follows:

$$
\int_{a}^{b} f^{\sigma}(t) g(t) \Delta t \geq \int_{c-\lambda_{1}}^{c+\lambda_{2}} f^{\sigma}(t) h(t) \Delta t
$$

Corollary 14. Setting $\alpha=0$ in Theorem 10, we obtain the nabla version of inequality (28) as follows:

$$
\int_{a}^{b} f^{\sigma}(t) g(t) \nabla t \geq \int_{c-\lambda_{1}}^{c+\lambda_{2}} f^{\sigma}(t) h(t) \nabla t
$$

Corollary 15. If $\mathbb{T}=\mathbb{R}$ and $\phi(t)=t$ in Corollary 13 , then, with the help of relations (1), we recapture [1] (Theorem 17 ).

Corollary 16. If $\mathbb{T}=\mathbb{Z}$ in Corollary 13, then, with the help of relations (2), inequality (29) becomes

$$
\sum_{t=a}^{b-1} f(t+1) g(t) \geq \sum_{t=c-\lambda_{1}}^{c+\lambda_{2}-1} f(t+1) h(t) .
$$

Theorem 11. Let $h:[a, b]_{\mathbb{T}} \rightarrow \mathbb{R}$ be a positive $\diamond_{\alpha}$-integrable function, let $f:[a, b]_{\mathbb{T}} \rightarrow \mathbb{R}$ be a $\nabla_{\alpha}$-integrable function, and let $c \in(a, b)$. Let $g, k:[a, b]_{\mathbb{T}} \rightarrow \mathbb{R}$ be $a \diamond_{\alpha}$-integrable function such that $0 \leq g(t) \leq k(t)$ for all $t \in[a, b]_{\mathbb{T}}$. Let $\lambda_{1}$ be the solution of the equation

$$
\int_{a}^{a+\lambda_{1}} k(t) h(t) \diamond_{\alpha} t=\int_{a}^{c} h(t) g(t) \diamond_{\alpha} t
$$

and let $\lambda_{2}$ be the solution of the equation

$$
\int_{b-\lambda_{2}}^{b} k(t) h(t) \diamond_{\alpha} t=\int_{c}^{b} h(t) g(t) \diamond_{\alpha} t
$$


If $f^{\sigma} / h \in \mathbb{A H}_{1}^{c}[a, b]$ and

$$
\int_{a}^{b} \phi(t) h(t) g(t) \diamond_{\alpha} t=\int_{a}^{a+\lambda_{1}} \phi(t) k(t) h(t) \diamond_{\alpha} t+\int_{b-\lambda_{2}}^{b} \phi(t) k(t) h(t) \diamond_{\alpha} t,
$$

then

$$
\int_{a}^{b} f^{\sigma}(t) g(t) \diamond_{\alpha} t \leq \int_{a}^{a+\lambda_{1}} f^{\sigma}(t) k(t) \diamond_{\alpha} t+\int_{b-\lambda_{2}}^{b} f^{\sigma}(t) k(t) \diamond_{\alpha} t .
$$

If $f^{\sigma} / h \in \mathbb{A H}_{2}^{c}[a, b]$ and (32) holds, the inequality in (33) is reversed.

Proof. Take $h \mapsto k h, g \mapsto g / k$ and $f \mapsto f k$ in Theorem 7 .

Corollary 17. Setting $\alpha=1$ in Theorem 11, we obtain the delta version of inequality (33) as follows:

$$
\int_{a}^{b} f^{\sigma}(t) g(t) \Delta t \leq \int_{a}^{a+\lambda_{1}} f^{\sigma}(t) k(t) \Delta t+\int_{b-\lambda_{2}}^{b} f^{\sigma}(t) k(t) \Delta t .
$$

Corollary 18. Setting $\alpha=0$ in Theorem 11, we obtain the nabla version of inequality (33) as follows:

$$
\int_{a}^{b} f^{\sigma}(t) g(t) \nabla t \leq \int_{a}^{a+\lambda_{1}} f^{\sigma}(t) k(t) \nabla t+\int_{b-\lambda_{2}}^{b} f^{\sigma}(t) k(t) \nabla t .
$$

Corollary 19. If $\mathbb{T}=\mathbb{R}$ and $\phi(t)=t$ in Corollary 17 , then, with the help of relations (1), we recapture [1] (Theorem 18 ).

Corollary 20. If $\mathbb{T}=\mathbb{Z}$ in Corollary 17, then, with the help of relations (2), inequality (34) becomes

$$
\sum_{t=a}^{b-1} f(t+1) g(t) \leq \sum_{t=a}^{a+\lambda_{1}-1} f(t+1) k(t)+\sum_{t=b-\lambda_{2}}^{b-1} f(t+1) k(t) .
$$

Theorem 12. Let $h:[a, b]_{\mathbb{T}} \rightarrow \mathbb{R}$ be a positive $\diamond_{\alpha}$-integrable function, let $f:[a, b]_{\mathbb{T}} \rightarrow \mathbb{R}$ be a $\diamond_{\alpha}$-integrable function, and let $c \in(a, b)$. Let $g, k:[a, b]_{\mathbb{T}} \rightarrow \mathbb{R}$ be $a \diamond_{\alpha}$-integrable function such that $0 \leq g(t) \leq k(t)$ for all $t \in[a, b]_{\mathbb{T}}$. Let $\lambda_{1}$ be the solution of the equation

$$
\int_{c-\lambda_{1}}^{c} k(t) h(t) \nabla_{\alpha} t=\int_{a}^{c} h(t) g(t) \nabla_{\alpha} t
$$

and let $\lambda_{2}$ be the solution of the equation

$$
\int_{\mathcal{c}}^{c+\lambda_{2}} k(t) h(t) \nabla_{\alpha} t=\int_{\mathcal{c}}^{b} h(t) g(t) \nabla_{\alpha} t
$$

If $f^{\sigma} / h \in \mathbb{A H}_{1}^{c}[a, b]$ and

$$
\int_{a}^{b} \phi(t) h(t) g(t) \diamond_{\alpha} t=\int_{\mathcal{c}-\lambda_{1}}^{c+\lambda_{2}} \phi(t) k(t) h(t) \diamond_{\alpha} t
$$

then

$$
\int_{a}^{b} f^{\sigma}(t) g(t) \diamond_{\alpha} t \geq \int_{c-\lambda_{1}}^{c+\lambda_{2}} f^{\sigma}(t) k(t) \diamond_{\alpha} t .
$$

If $f^{\sigma} / h \in \mathbb{A H}_{2}^{c}[a, b]$ and (37) holds, the inequality in (38) is reversed.

Proof. Take $h \mapsto k h, g \mapsto g / k$ and $f \mapsto f k$ in Theorem 8 . 
Corollary 21. Setting $\alpha=1$ in Theorem 12, we obtain the delta version of inequality (38) as follows:

$$
\int_{a}^{b} f^{\sigma}(t) g(t) \Delta t \geq \int_{c-\lambda_{1}}^{c+\lambda_{2}} f^{\sigma}(t) k(t) \Delta t
$$

Corollary 22. Setting $\alpha=0$ in Theorem 12, we obtain the nabla version of inequality (38) as follows:

$$
\int_{a}^{b} f^{\sigma}(t) g(t) \nabla t \geq \int_{c-\lambda_{1}}^{c+\lambda_{2}} f^{\sigma}(t) k(t) \nabla t
$$

Corollary 23. If $\mathbb{T}=\mathbb{R}$ and $\phi(t)=t$ in Corollary 21, then, with the help of relations (1), we recapture [1] (Theorem 19).

Corollary 24. If $\mathbb{T}=\mathbb{Z}$ in Corollary 21, then, with the help of relations (2), inequality (39) becomes

$$
\sum_{t=a}^{b-1} f(t+1) g(t) \geq \sum_{t=c-\lambda_{1}}^{c+\lambda_{2}-1} f(t+1) k(t) .
$$

Theorem 13. Let $h:[a, b]_{\mathbb{T}} \rightarrow \mathbb{R}$ be a positive $\diamond_{\alpha}$-integrable function, let $f:[a, b]_{\mathbb{T}} \rightarrow \mathbb{R}$ be $a$ $\nabla_{\alpha}$-integrable function, and let $c \in(a, b)$. Let $g:[a, b]_{\mathbb{T}} \rightarrow \mathbb{R}$ be $a \diamond_{\alpha}$-integrable function such that $0 \leq g(t) \leq 1$ for all $t \in[a, b]_{\mathbb{T}}$. Let $\lambda_{1}$ be the solution of the equation

$$
\int_{a}^{a+\lambda_{1}} h(t) \nabla_{\alpha} t=\int_{a}^{c} h(t) g(t) \diamond_{\alpha} t,
$$

and let $\lambda_{2}$ be the solution of the equation

$$
\int_{b-\lambda_{2}}^{b} h(t) \nabla_{\alpha} t=\int_{c}^{b} h(t) g(t) \diamond_{\alpha} t .
$$

If $f^{\sigma} / h \in \mathbb{A H}_{1}^{c}[a, b]$ and

$$
\begin{aligned}
& \int_{a}^{b} \phi(t) h(t) g(t) \nabla_{\alpha} t \\
& =\int_{a}^{a+\lambda_{1}}\left(\phi(t) h(t)-\left[\phi(t)-a-\lambda_{1}\right] h(t)[1-g(t)]\right) \nabla_{\alpha} t \\
& \quad+\int_{b-\lambda_{2}}^{b}\left(\phi(t) h(t)-\left[\phi(t)-b+\lambda_{2}\right] h(t)[1-g(t)]\right) \nabla_{\alpha} t
\end{aligned}
$$

then

$$
\begin{aligned}
& \int_{a}^{b} f^{\sigma}(t) g(t) \diamond_{\alpha} t \\
& \leq \int_{a}^{a+\lambda_{1}}\left(f^{\sigma}(t)-\left[\frac{f^{\sigma}(t)}{h(t)}-\frac{f^{\sigma}\left(a+\lambda_{1}\right)}{h\left(a+\lambda_{1}\right)}\right] h(t)[1-g(t)]\right) \nabla_{\alpha} t \\
& \quad+\int_{b-\lambda_{2}}^{b}\left(f^{\sigma}(t)-\left[\frac{f^{\sigma}(t)}{h(t)}-\frac{f^{\sigma}\left(b-\lambda_{2}\right)}{h\left(b-\lambda_{2}\right)}\right] h(t)[1-g(t)]\right) \nabla_{\alpha} t .
\end{aligned}
$$

If $f^{\sigma} / h \in \mathbb{A H}_{2}^{c}[a, b]$ and (42) holds, the inequality in (43) is reversed.

Proof. The proof is similar to that of Theorem 7 applying Lemma 4 for $F / h:[a, c]_{\mathbb{T}} \rightarrow \mathbb{R}$ nonincreasing and for $F / h:[c, b]_{\mathbb{T}} \rightarrow \mathbb{R}$ nondecreasing. 
Corollary 25. Setting $\alpha=1$ in Theorem 13, we obtain the delta version of inequality (43) as follows:

$$
\begin{aligned}
& \int_{a}^{b} f^{\sigma}(t) g(t) \Delta t \\
& \leq \int_{a}^{a+\lambda_{1}}\left(f^{\sigma}(t)-\left[\frac{f^{\sigma}(t)}{h(t)}-\frac{f^{\sigma}\left(a+\lambda_{1}\right)}{h\left(a+\lambda_{1}\right)}\right] h(t)[1-g(t)]\right) \Delta t \\
& \quad+\int_{b-\lambda_{2}}^{b}\left(f^{\sigma}(t)-\left[\frac{f^{\sigma}(t)}{h(t)}-\frac{f^{\sigma}\left(b-\lambda_{2}\right)}{h\left(b-\lambda_{2}\right)}\right] h(t)[1-g(t)]\right) \Delta t
\end{aligned}
$$

Corollary 26. Setting $\alpha=0$ in Theorem 13, we obtain the nabla version of inequality (43) as follows:

$$
\begin{aligned}
& \int_{a}^{b} f^{\sigma}(t) g(t) \nabla t \\
& \leq \int_{a}^{a+\lambda_{1}}\left(f^{\sigma}(t)-\left[\frac{f^{\sigma}(t)}{h(t)}-\frac{f^{\sigma}\left(a+\lambda_{1}\right)}{h\left(a+\lambda_{1}\right)}\right] h(t)[1-g(t)]\right) \nabla t \\
& \quad+\int_{b-\lambda_{2}}^{b}\left(f^{\sigma}(t)-\left[\frac{f^{\sigma}(t)}{h(t)}-\frac{f^{\sigma}\left(b-\lambda_{2}\right)}{h\left(b-\lambda_{2}\right)}\right] h(t)[1-g(t)]\right) \nabla t
\end{aligned}
$$

Corollary 27. If $\mathbb{T}=\mathbb{R}$ and $\phi(t)=t$ in Corollary 25, then, with the help of relations (1), we recapture [1] (Theorem 21 ).

Corollary 28. If $\mathbb{T}=\mathbb{Z}$ in Corollary 25, then, with the help of relations (2), inequality (44) becomes

$$
\begin{aligned}
& \sum_{t=a}^{b-1} f(t+1) g(t) \\
& \leq \sum_{t=a}^{a+\lambda_{1}-1}\left(f(t+1)-\left[\frac{f(t+1)}{h(t)}-\frac{f\left(a+\lambda_{1}+1\right)}{h\left(a+\lambda_{1}\right)}\right] h(t)[1-g(t)]\right) \\
& \quad+\sum_{t=b-\lambda_{2}}^{b-1}\left(f(t+1)-\left[\frac{f(t+1)}{h(t)}-\frac{f\left(b-\lambda_{2}+1\right)}{h\left(b-\lambda_{2}\right)}\right] h(t)[1-g(t)]\right) .
\end{aligned}
$$

Theorem 14. Let $h:[a, b]_{\mathbb{T}} \rightarrow \mathbb{R}$ be a positive $\diamond_{\alpha}$-integrable function, let $f:[a, b]_{\mathbb{T}} \rightarrow \mathbb{R}$ be $a$ $\diamond_{\alpha}$-integrable function, and let $c \in(a, b)$. Let $g:[a, b]_{\mathbb{T}} \rightarrow \mathbb{R}$ be $a \diamond_{\alpha}$-integrable function such that $0 \leq g(t) \leq 1$ for all $t \in[a, b]_{\mathbb{T}}$. Let $\lambda_{1}$ be the solution of the equation

$$
\int_{c-\lambda_{1}}^{c} h(t) \nabla_{\alpha} t=\int_{a}^{c} h(t) g(t) \diamond_{\alpha} t,
$$

and let $\lambda_{2}$ be the solution of the equation

$$
\int_{c}^{c+\lambda_{2}} h(t) \diamond_{\alpha} t=\int_{c}^{b} h(t) g(t) \diamond_{\alpha} t .
$$

If $f^{\sigma} / h \in \mathbb{A H}_{1}^{c}[a, b]$ and

$$
\begin{aligned}
& \int_{a}^{b} \phi(t) h(t) g(t) \nabla_{\alpha} t \\
& =\int_{c-\lambda_{1}}^{c}\left(\phi(t) h(t)-\left[\phi(t)-c+\lambda_{1}\right] h(t)[1-g(t)]\right) \nabla_{\alpha} t \\
& =\int_{a}^{a+\lambda_{1}}\left(\phi(t) h(t)-\left[\phi(t)-c+\lambda_{2}\right] h(t)[1-g(t)]\right) \nabla_{\alpha} t
\end{aligned}
$$


then

$$
\begin{aligned}
& \int_{a}^{b} f^{\sigma}(t) g(t) \nabla_{\alpha} t \\
& \geq \int_{c-\lambda_{1}}^{c}\left(f^{\sigma}(t)-\left[\frac{f^{\sigma}(t)}{h(t)}-\frac{f^{\sigma}\left(c-\lambda_{1}\right)}{h\left(c-\lambda_{1}\right)}\right] h(t)[1-g(t)]\right) \nabla_{\alpha} t \\
& \quad+\int_{c}^{c+\lambda_{2}}\left(f^{\sigma}(t)-\left[\frac{f^{\sigma}(t)}{h(t)}-\frac{f^{\sigma}\left(c+\lambda_{2}\right)}{h\left(c+\lambda_{2}\right)}\right] h(t)[1-g(t)]\right) \nabla_{\alpha} t .
\end{aligned}
$$

If $f^{\sigma} / h \in \mathbb{A H}_{2}^{c}[a, b]$ and (47) holds, the inequality in (48) is reversed.

Proof. The proof is similar to that of Theorem 8 applying Lemma 4 for $F / h:[a, c]_{\mathbb{T}} \rightarrow \mathbb{R}$ nonincreasing and for $F / h:[c, b]_{\mathbb{T}} \rightarrow \mathbb{R}$ nondecreasing.

Corollary 29. Setting $\alpha=1$ in Theorem 14, we obtain the delta version of inequality (48) as follows:

$$
\begin{aligned}
& \int_{a}^{b} f^{\sigma}(t) g(t) \Delta t \\
& \geq \int_{c-\lambda_{1}}^{c}\left(f^{\sigma}(t)-\left[\frac{f^{\sigma}(t)}{h(t)}-\frac{f^{\sigma}\left(c-\lambda_{1}\right)}{h\left(c-\lambda_{1}\right)}\right] h(t)[1-g(t)]\right) \Delta t \\
& \quad+\int_{c}^{c+\lambda_{2}}\left(f^{\sigma}(t)-\left[\frac{f^{\sigma}(t)}{h(t)}-\frac{f^{\sigma}\left(c+\lambda_{2}\right)}{h\left(c+\lambda_{2}\right)}\right] h(t)[1-g(t)]\right) \Delta t .
\end{aligned}
$$

Corollary 30. Setting $\alpha=0$ in Theorem 14, we obtain the nabla version of inequality (48) as follows:

$$
\begin{aligned}
& \int_{a}^{b} f^{\sigma}(t) g(t) \nabla t \\
& \geq \int_{c-\lambda_{1}}^{c}\left(f^{\sigma}(t)-\left[\frac{f^{\sigma}(t)}{h(t)}-\frac{f^{\sigma}\left(c-\lambda_{1}\right)}{h\left(c-\lambda_{1}\right)}\right] h(t)[1-g(t)]\right) \nabla t \\
& \quad+\int_{c}^{c+\lambda_{2}}\left(f^{\sigma}(t)-\left[\frac{f^{\sigma}(t)}{h(t)}-\frac{f^{\sigma}\left(c+\lambda_{2}\right)}{h\left(c+\lambda_{2}\right)}\right] h(t)[1-g(t)]\right) \nabla t .
\end{aligned}
$$

Corollary 31. If $\mathbb{T}=\mathbb{R}$ and $\phi(t)=t$ in Corollary 29, then, with the help of relations (1), we recapture [1] (Theorem 22 ).

Corollary 32. If $\mathbb{T}=\mathbb{Z}$ in Corollary 29, then, with the help of relations (2), inequality (49) becomes

$$
\begin{aligned}
& \sum_{t=a}^{b-1} f(t+1) g(t) \\
& \geq \sum_{t=c-\lambda_{1}}^{c-1}\left(f(t+1)-\left[\frac{f(t+1)}{h(t)}-\frac{f\left(c-\lambda_{1}+1\right)}{h\left(c-\lambda_{1}\right)}\right] h(t)[1-g(t)]\right) \\
& \quad+\sum_{t=c}^{c+\lambda_{2}-1}\left(f(t+1)-\left[\frac{f(t+1)}{h(t)}-\frac{f\left(c+\lambda_{2}+1\right)}{h\left(c+\lambda_{2}\right)}\right] h(t)[1-g(t)]\right) .
\end{aligned}
$$

Theorem 15. Let $h:[a, b]_{\mathbb{T}} \rightarrow \mathbb{R}$ be a positive $\diamond_{\alpha}$-integrable function, let $f:[a, b]_{\mathbb{T}} \rightarrow \mathbb{R}$ be $a$ $\nabla_{\alpha}$-integrable function, and let $c \in(a, b)$. Let $g, \psi:[a, b]_{\mathbb{T}} \rightarrow \mathbb{R}$ be $a \diamond_{\alpha}$-integrable function such that $0 \leq \psi(t) \leq g(t) \leq 1-\psi(t)$ for all $t \in[a, b]_{\mathbb{T}}$. Let $\lambda_{1}$ be the solution of the equation

$$
\int_{a}^{a+\lambda_{1}} h(t) \diamond_{\alpha} t=\int_{a}^{c} h(t) g(t) \diamond_{\alpha} t
$$


and let $\lambda_{2}$ be the solution of the equation

$$
\int_{b-\lambda_{2}}^{b} h(t) \diamond_{\alpha} t=\int_{c}^{b} h(t) g(t) \diamond_{\alpha} t .
$$

If $f^{\sigma} / h \in \mathbb{A H}_{1}^{c}[a, b]$ and

$$
\begin{aligned}
& \int_{a}^{b} \phi(t) h(t) g(t) \diamond_{\alpha} t \\
& =\int_{a}^{a+\lambda_{1}} \phi(t) h(t) \diamond_{\alpha} t-\int_{a}^{c}\left|\phi(t)-a-\lambda_{1}\right| h(t) \psi(t) \diamond_{\alpha} t+\int_{b-\lambda_{2}}^{b} \phi(t) h(t) \diamond_{\alpha} t \\
& \quad+\int_{c}^{b}\left|\phi(t)-b+\lambda_{2}\right| h(t) \psi(t) \diamond_{\alpha} t,
\end{aligned}
$$

then

$$
\begin{aligned}
& \int_{a}^{b} f^{\sigma}(t) g(t) \diamond_{\alpha} t \\
& \leq \int_{a}^{a+\lambda_{1}} f^{\sigma}(t) \diamond_{\alpha} t-\int_{a}^{c}\left|\frac{f^{\sigma}(t)}{h(t)}-\frac{f^{\sigma}\left(a+\lambda_{1}\right)}{h\left(a+\lambda_{1}\right)}\right| h(t) \psi(t) \diamond_{\alpha} t+\int_{b-\lambda_{2}}^{b} f^{\sigma}(t) \diamond_{\alpha} t \\
& +\int_{c}^{b}\left|\frac{f^{\sigma}(t)}{h(t)}-\frac{f^{\sigma}\left(b-\lambda_{2}\right)}{h\left(b-\lambda_{2}\right)}\right| h(t) \psi(t) \nabla_{\alpha} t .
\end{aligned}
$$

If $f^{\sigma} / h \in \mathbb{A H}_{2}^{c}[a, b]$ and (52) holds, the inequality in (53) is reversed.

Proof. The proof is similar to that of Theorem 7 applying Lemma 3 for $F / h:[a, c]_{\mathbb{T}} \rightarrow \mathbb{R}$ nonincreasing and Lemma 3 for $F / h:[c, b]_{\mathbb{T}} \rightarrow \mathbb{R}$ nondecreasing.

Corollary 33. Setting $\alpha=1$ in Theorem 15, we obtain the delta version of inequality (53) as follows:

$$
\begin{aligned}
& \int_{a}^{b} f^{\sigma}(t) g(t) \Delta t \\
& \leq \int_{a}^{a+\lambda_{1}} f^{\sigma}(t) \Delta t-\int_{a}^{c}\left|\frac{f^{\sigma}(t)}{h(t)}-\frac{f^{\sigma}\left(a+\lambda_{1}\right)}{h\left(a+\lambda_{1}\right)}\right| h(t) \psi(t) \Delta t+\int_{b-\lambda_{2}}^{b} f^{\sigma}(t) \Delta t \\
& +\int_{c}^{b}\left|\frac{f^{\sigma}(t)}{h(t)}-\frac{f^{\sigma}\left(b-\lambda_{2}\right)}{h\left(b-\lambda_{2}\right)}\right| h(t) \psi(t) \Delta t .
\end{aligned}
$$

Corollary 34. Setting $\alpha=0$ in Theorem 15, we obtain the nabla version of inequality (53) as follows:

$$
\begin{aligned}
& \int_{a}^{b} f^{\sigma}(t) g(t) \nabla t \\
& \leq \int_{a}^{a+\lambda_{1}} f^{\sigma}(t) \nabla t-\int_{a}^{c}\left|\frac{f^{\sigma}(t)}{h(t)}-\frac{f^{\sigma}\left(a+\lambda_{1}\right)}{h\left(a+\lambda_{1}\right)}\right| h(t) \psi(t) \nabla t+\int_{b-\lambda_{2}}^{b} f^{\sigma}(t) \nabla t \\
& +\int_{c}^{b}\left|\frac{f^{\sigma}(t)}{h(t)}-\frac{f^{\sigma}\left(b-\lambda_{2}\right)}{h\left(b-\lambda_{2}\right)}\right| h(t) \psi(t) \nabla t .
\end{aligned}
$$

Corollary 35. If $\mathbb{T}=\mathbb{R}$ and $\phi(t)=t$ in Corollary 33, then, with the help of relations (1), we recapture [1] (Theorem 23). 
Corollary 36. If $\mathbb{T}=\mathbb{Z}$ in Corollary 33, then, with the help of relations (2), inequality (54) becomes

$$
\begin{aligned}
& \sum_{t=a}^{b-1} f(t+1) g(t) \\
& \leq \sum_{t=a}^{a+\lambda_{1}-1} f(t+1)-\sum_{t=a}^{c-1}\left|\frac{f(t+1)}{h(t)}-\frac{f\left(a+\lambda_{1}+1\right)}{h\left(a+\lambda_{1}\right)}\right| h(t) \psi(t) \nabla t+\sum_{t=b-\lambda_{2}}^{b-1} f(t+1) \\
& \quad+\sum_{t=c}^{b-1}\left|\frac{f(t+1)}{h(t)}-\frac{f\left(b-\lambda_{2}+1\right)}{h\left(b-\lambda_{2}\right)}\right| h(t) \psi(t) .
\end{aligned}
$$

Theorem 16. Let $h:[a, b]_{\mathbb{T}} \rightarrow \mathbb{R}$ be a positive $\diamond_{\alpha}$-integrable function, let $f:[a, b]_{\mathbb{T}} \rightarrow \mathbb{R}$ be $a$ $\diamond_{\alpha}$-integrable function, and let $c \in(a, b)$. Let $g, \psi:[a, b]_{\mathbb{T}} \rightarrow \mathbb{R}$ be $a \diamond_{\alpha}$-integrable function such that $0 \leq \psi(t) \leq g(t) \leq 1-\psi(t)$ for all $t \in[a, b]_{\mathbb{T}}$. Let $\lambda_{1}$ be the solution of the equation

$$
\int_{c-\lambda_{1}}^{c} h(t) \diamond_{\alpha} t=\int_{a}^{c} h(t) g(t) \diamond_{\alpha} t
$$

and let $\lambda_{2}$ be the solution of the equation

$$
\int_{c}^{c+\lambda_{2}} h(t) \nabla_{\alpha} t=\int_{c}^{b} h(t) g(t) \nabla_{\alpha} t
$$

If $f^{\sigma} / h \in \mathbb{A H}_{1}^{c}[a, b]$ and

$$
\int_{a}^{b} \phi(t) h(t) g(t) \diamond_{\alpha} t=\int_{c-\lambda_{1}}^{c} \phi(t) h(t) \diamond_{\alpha} t-\int_{a}^{c}\left|\phi(t)-c+\lambda_{1}\right| h(t) \psi(t) \diamond_{\alpha} t+\int_{c}^{b}\left|\phi(t)-c-\lambda_{1}\right| h(t) \psi(t) \diamond_{\alpha} t
$$

then

$$
\begin{aligned}
& \int_{a}^{b} f^{\sigma}(t) g(t) \diamond_{\alpha} t \\
& \geq \int_{c-\lambda_{1}}^{c+\lambda_{2}} f^{\sigma}(t) \diamond_{\alpha} t+\int_{a}^{c}\left|\frac{f^{\sigma}(t)}{h(t)}-\frac{f^{\sigma}\left(c-\lambda_{1}\right)}{h\left(c-\lambda_{1}\right)}\right| h(t) \psi(t) \diamond_{\alpha} t \\
& \quad-\int_{c}^{b}\left|\frac{f^{\sigma}(t)}{h(t)}-\frac{f^{\sigma}\left(c+\lambda_{2}\right)}{h\left(c+\lambda_{2}\right)}\right| h(t) \psi(t) \diamond_{\alpha} t .
\end{aligned}
$$

If $f^{\sigma} / h \in \mathbb{A H}_{2}^{c}[a, b]$ and (57) holds, the inequality in (58) is reversed.

Proof. The proof is similar to that of Theorem 8 applying Lemma 3 for $F / h:[a, c]_{\mathbb{T}} \rightarrow \mathbb{R}$ nonincreasing and Lemma 3 for $F / h:[c, b]_{\mathbb{T}} \rightarrow \mathbb{R}$ nondecreasing.

Corollary 37. Setting $\alpha=1$ in Theorem 16, we obtain the delta version of inequality (58) as follows:

$$
\begin{aligned}
& \int_{a}^{b} f^{\sigma}(t) g(t) \Delta t \\
& \geq \int_{c-\lambda_{1}}^{c+\lambda_{2}} f^{\sigma}(t) \Delta t+\int_{a}^{c}\left|\frac{f^{\sigma}(t)}{h(t)}-\frac{f^{\sigma}\left(c-\lambda_{1}\right)}{h\left(c-\lambda_{1}\right)}\right| h(t) \psi(t) \Delta t \\
& \quad-\int_{c}^{b}\left|\frac{f^{\sigma}(t)}{h(t)}-\frac{f^{\sigma}\left(c+\lambda_{2}\right)}{h\left(c+\lambda_{2}\right)}\right| h(t) \psi(t) \Delta t .
\end{aligned}
$$


Corollary 38. Setting $\alpha=0$ in Theorem 16, we obtain the nabla version of inequality (58) as follows:

$$
\begin{aligned}
& \int_{a}^{b} f^{\sigma}(t) g(t) \nabla t \\
& \geq \int_{c-\lambda_{1}}^{c+\lambda_{2}} f^{\sigma}(t) \nabla t+\int_{a}^{c}\left|\frac{f^{\sigma}(t)}{h(t)}-\frac{f^{\sigma}\left(c-\lambda_{1}\right)}{h\left(c-\lambda_{1}\right)}\right| h(t) \psi(t) \nabla t \\
& \quad-\int_{c}^{b}\left|\frac{f^{\sigma}(t)}{h(t)}-\frac{f^{\sigma}\left(c+\lambda_{2}\right)}{h\left(c+\lambda_{2}\right)}\right| h(t) \psi(t) \nabla t
\end{aligned}
$$

Corollary 39. If $\mathbb{T}=\mathbb{R}$ and $\phi(t)=t$ in Corollary 37, then, with the help of relations (1), we recapture [1] (Theorem 24 ).

Corollary 40. If $\mathbb{T}=\mathbb{Z}$ in Corollary 37, then, with the help of relations (2), inequality (59 becomes

$$
\begin{aligned}
& \sum_{t=a}^{b-1} f(t+1) g(t) \\
& \geq \sum_{t=c-\lambda_{1}}^{c+\lambda_{2}-1} f(t+1)+\sum_{t=a}^{c-1}\left|\frac{f(t+1)}{h(t)}-\frac{f\left(c-\lambda_{1}+1\right)}{h\left(c-\lambda_{1}\right)}\right| h(t) \psi(t) \\
& \quad-\sum_{t=c}^{b-1}\left|\frac{f(t+1)}{h(t)}-\frac{f\left(c+\lambda_{2}+1\right)}{h\left(c+\lambda_{2}\right)}\right| h(t) \psi(t) .
\end{aligned}
$$

\section{Conclusions}

In this important work, we have discussed some new dynamic inequalities of the Steffensen-type by using diamond-alpha integral on time scales. We further presented some relevant inequalities as special cases: discrete inequalities and integral inequalities. These results may possibly be used to obtain more generalized results of several previously obtained inequalities by setting $\alpha=0$ and $\alpha=1$.

Author Contributions: Conceptualization, resources and methodology, A.A.E.-D. and O.B.; investigation, supervision, J.A.; data curation, O.B.; writing-original draft preparation, A.A.E.-D.; writing-review and editing, J.A.; project administration, A.A.E.-D. and O.B. All authors read and agreed to the published version of the manuscript.

Funding: This research received no external funding.

Institutional Review Board Statement: Not applicable.

Informed Consent Statement: Not applicable.

Data Availability Statement: Not applicable.

Conflicts of Interest: The authors declare no conflict of interest.

\section{References}

1. Pećarixcx, J.; Kalamir, K.S. Generalized Steffensen type inequalities involving convex functions. J. Funct. Spaces 2014, 10, 428030.

2. Hilger, S. Analysis on measure chains-a unified approach to continuous and discrete calculus. Results Math. 1990, 18, 18-56. [CrossRef]

3. Hilger, S. Ein Maßkettenkalkül Mit Anwendung Auf ZEntrumsmannigfaltigkeiten. Ph.D. Thesis, Universität Würzburg, Würzburg, Germany, 1988.

4. Bohner, M.; Peterson, A. Dynamic Equations on Time Scales: An Introduction with Applications; Birkhäuser Boston, Inc.: Boston, MA, USA, 2001.

5. Sheng, Q.; Fadag, M.; Henderson, J.; Davis, J.M. An exploration of combined dynamic derivatives on time scales and their applications. Nonlinear Anal. Real World Appl. 2006, 7, 395-413. [CrossRef]

6. Abdeldaim, A.; El-Deeb, A.A.; Agarwal, P.; El-Sennary, H.A. On some dynamic inequalities of Steffensen type on time scales. Math. Methods Appl. Sci. 2018, 41, 4737-4753. [CrossRef] 
7. Agarwal, R.; Bohner, M.; Peterson, A. Inequalities on time scales: a survey. Math. Inequal. Appl. 2001, 4, 535-557. [CrossRef]

8. Agarwal, R.; O’Regan, D.; Saker, S. Dynamic Inequalities on Time Scales; Springer: Cham, Swizerland, 2014.

9. El-Deeb, A.A. A variety of nonlinear retarded integral inequalities of gronwall type and their applications. In Advances in Mathematical Inequalities and Applications; Springer: Berlin/Heidelberg, Germany, 2018; pp. 143-164.

10. El-Deeb, A.A.; El-Sennary, H.A.; Khan, Z.A. Some Steffensen-type dynamic inequalities on time scales. Adv. Differ. Equ. 2019, 2019, 246 . [CrossRef]

11. Li, W.N. Some new dynamic inequalities on time scales. J. Math. Anal. Appl. 2006, 319, 802-814. [CrossRef]

12. Anastassiou, G.A. Foundations of nabla fractional calculus on time scales and inequalities. Comput. Math. Appl. 2010, 59, 3750-3762. [CrossRef]

13. Anastassiou, G.A. Principles of delta fractional calculus on time scales and inequalities. Math. Comput. Model. 2010, 52, 556-566. [CrossRef]

14. Anastassiou, G.A. Integral operator inequalities on time scales. Int. J. Differ. Equ. 2012, 7, 111-137.

15. Sahir, M. Dynamic inequalities for convex functions harmonized on time scales. J. App. Math. Phys. 2017, 5, 2360-2370. [CrossRef]

16. Anderson, D.R. Time-scale integral inequalities. JIPAM. J. Inequal. Pure Appl. Math. 2005, 6, 66.

17. Ozkan, U.M.; Yildirim, H. Steffensen's integral inequality on time scales. J. Inequal. Appl. 2007, 2007, 46524. [CrossRef] 